\title{
Prevention, Detection, and Management of Heart Failure in Patients Treated for Breast Cancer
}

\author{
Agneta Månsson Broberg ${ }^{1,2}$. Jürgen Geisler ${ }^{3}$. Suvi Tuohinen ${ }^{4} \cdot$ Tanja Skytta $^{5}$ • Pórdís Jóna Hrafnkelsdóttir ${ }^{6}$. \\ Kirsten Melgaard Nielsen ${ }^{7}$. Elham Hedayati ${ }^{8,9}$. Torbjørn Omland ${ }^{10}$ • Birgitte V. Offersen ${ }^{11}$. Alexander R. Lyon ${ }^{12}$. \\ Geeta Gulati $^{13,14}$
}

Accepted: 8 September 2020 / Published online: 26 September 2020

(C) The Author(s) 2020

\begin{abstract}
Purpose of Review Long-term survival has increased significantly in breast cancer patients, and cardiovascular side effects are surpassing cancer-related mortality. We summarize risk factors, prevention strategies, detection, and management of cardiotoxicity, with focus on left ventricular dysfunction and heart failure, during breast cancer treatment.

Recent Findings Baseline treatment of cardiovascular risk factors is recommended. Anthracycline and trastuzumab treatment constitute a substantial risk of developing cardiotoxicity. There is growing evidence that this can be treated with beta blockers and angiotensin antagonists. Early detection of cardiotoxicity with cardiac imaging and circulating cardiovascular biomarkers is currently evaluated in clinical trials. Chest wall irradiation accelerates atherosclerotic processes and induces fibrosis. Immune checkpoint inhibitors require consideration for surveillance due to a small risk of severe myocarditis. Cyclin-dependent kinases4/6 inhibitors, cyclophosphamide, taxanes, tyrosine kinase inhibitors, and endocrine therapy have a lower-risk profile for cardiotoxicity.

Summary Preventive and management strategies to counteract cancer treatment-related left ventricular dysfunction or heart failure in breast cancer patients should include a comprehensive cardiovascular risk assessment and individual clinical evaluation. This should include both patient and treatment-related factors. Further clinical trials especially on early detection, cardioprevention, and management are urgently needed.
\end{abstract}

Keywords Breast cancer $\cdot$ Cardiotoxicity $\cdot$ Heart failure $\cdot$ Trastuzumab $\cdot$ Anthracyclines $\cdot$ Radiation therapy

\section{Introduction}

Breast cancer is the leading cause of cancer in women worldwide with a mean age standardized incidence of 46.3/100000,

This article is part of the Topical Collection on Cardio-Oncology

Geeta Gulati

geetagul@medisin.uio.no

Agneta Månsson Broberg

agneta.mansson.broberg@ki.se

Jürgen Geisler

juergen.geisler@medisin.uio.no

Suvi Tuohinen

suvi.tuohinen@hus.fi

Tanja Skytta

tanja.skytta@fimnet.fi

Pórdís Jóna Hrafnkelsdóttir

thordisj@landspitali.is ranging from 25.9 in South East Asia to 94.2 in Australia and New Zeeland [1]. While the incidence is slightly increasing, the mortality is decreasing, likely due to the effective screening programs and evolving treatment.

\author{
Kirsten Melgaard Nielsen \\ kirstnls@rm.dk \\ Elham Hedayati \\ elham.hedayati@ki.se \\ Torbjørn Omland \\ torbjorn.omland@medisin.uio.no \\ Birgitte V. Offersen \\ bvo@oncology.au.dk \\ Alexander R. Lyon \\ A.Lyon@rbht.nhs.uk
}

Extended author information available on the last page of the article 
Breast cancer treatment is based on the individual tumor's expression of specific markers as estrogen receptor (ER), progesterone receptor (PR), human epidermal growth factor receptor (HER) 2,the proliferation marker Ki-67, and the extent of the disease. Combining surgery, chemotherapy, and radiation therapy with targeted therapy, the outcome has improved markedly over the past two decades and the average 5-year survival is currently approximately $87 \%$ [1]. The prolonged survival has led to an increased focus on the off-target-effects of breast cancer treatment. In fact, cardiovascular complications may surpass cancer-related mortality in a subgroup of patients [2]. Short-term acute left ventricular dysfunction, arrhythmia, or ischemic events can hinder patients from receiving life-saving cancer treatment. The patients are also at risk of developing long-term cardiovascular morbidity, especially decreased left ventricular function and heart failure. Heart failure is associated with reduced quality of life and increased mortality. In this article we summarize the clinically most relevant risk factors for cardiovascular complications during breast cancer therapy and discuss strategies for prevention, detection, and management of cardiotoxicity. We will focus on left ventricular dysfunction and heart failure.

\section{Commonly Used Chemotherapy}

Anthracyclines, i.e., epirubicin and doxorubicin, are among the most widely used conventional chemotherapeutics established in breast cancer treatment. Anthracyclines inhibit deoxyribonucleic acid (DNA) synthesis and alters DNA transcription and replication by intercalating DNA and ribonucleic acid (RNA) strands and inhibiting topoisomerase II $\alpha$ [3]. Anthracyclines also induce an iron-mediated oxidative stress that damages DNA, proteins, and lipids [4]. Cardiomyocytes and their mitochondria seem to be especially vulnerable to the damage via inhibition of topoisomerase II $\beta$ and increased oxidative stress [5]. Dysregulation of epigenomic and transcriptomic responses have also been reported [6]. Importantly, cardiotoxicity is a clinically dose-limiting adverse effect of anthracyclines. In a retrospective report including three randomized studies (doxorubicin/placebo), decompensated congestive heart failure increased from $4.7 \%$ at $400 \mathrm{mg} / \mathrm{m}^{2}$ doxorubicin to $48 \%$ at doses of $650-800 \mathrm{mg} / \mathrm{m}^{2}$ [7]. About $50 \%$ of these patients had an absolute left ventricular ejection fraction (LVEF) drop of more than $30 \%$. Importantly so, using sensitive echocardiographic methods such as global longitudinal strain (GLS), early signs of cardiotoxicity have been seen in doxorubicin doses as low as $150 \mathrm{mg} / \mathrm{m}^{2}$ [8]. Acute anthracycline-induced cardiotoxicity occurs within a week from treatment and is rare $(<5 \%)$. It resembles an acute toxic myocarditis with a histopathological cardiomyocyte damage, inflammatory infiltration, and interstitial edema [6,9]. Often electrocardiographic (ECG) changes are present with brady- or tachy-arrhythmias and clinical symptoms as palpitations, dyspnea, chest pain, heart failure, syncope, or cardiac arrest. Chronic anthracycline-induced cardiotoxicity is histopathological typically characterized by vacuole formation, necrosis, myofibril dropout, and fibrosis [9]. By echocardiography this can be seen as a drop in LVEF or GLS. A decline in LVEF of $\geq 10$ percentage to a value below $50 \%$ is widely accepted to be of significance [10]. Typical risk factors for anthracycline-induced cardiotoxicity are age $<5$ or $>65$ years, a family history of premature cardiovascular disease, arterial hypertension, diabetes mellitus, hypercholesterolemia, current myocardial disease, prior radiation to chest or mediastinum, smoking, and obesity [11, $12 \bullet \bullet$.

In conclusion, anthracyclines are a major cause of cancer treatment-related cardiotoxicity in breast cancer patients.

Cyclophosphamide is an alkylating agent that in breast cancer patients usually is combined with anthracyclines and taxanes in the adjuvant setting. The cardiotoxicity is described as ECG changes, left ventricular dysfunction, and heart failure. There is conflicting evidence about the incidence, and numbers vary from $<1$ to $28 \%$ [13]. In the standard adjuvant regimen for breast cancer $600 \mathrm{mg} / \mathrm{m}^{2}$, cyclophosphamide is given 3-4 times with 3-week interval. Recently, a doseescalating phase III trial tested intensified treatment with cyclophosphamide, in myeloma patients, with doses up to $500 \mathrm{mg}$ orally day $1,8,15$, and 3 weekly cycles until maximum response or intolerance. In total, 4 patients $(<1 \%)$ had cardiac disorders, and this was not further specified [14]. In conclusion, the results support the low-risk profile for cardiac morbidity using cyclophosphamide in modern breast cancer treatment.

Taxanes are agents inhibiting mitotic activity, and the most frequently used drugs are docetaxel and paclitaxel. Taxanes can be used as monotherapy or combined with other chemotherapeutics including trastuzumab and pertuzumab. Cardiotoxicity with taxanes is infrequent. Reports are conflicting as the cardiotoxic definition is inconsistent, including asymptomatic bradycardia to ischemic events [15]. Additionally, it has been debated whether concomitant use of paclitaxel and doxorubicin aggravate doxorubicin induced cardiotoxicity $[16,17]$. The incidence of docetaxel associated heart failure is $<2 \%[16,18]$. The incidence of myocardial ischemia has only been reported in the drugs summary of product characteristics (SPC) [19]. In a Cochrane review of taxanes for adjuvant treatment of early breast cancer twenty three studies included data on cardiotoxicity [20]. Pooled analysis showed small to no difference in cardiotoxicity of taxane compared with nontaxane-containing cancer treatment regimens (OR 0.87, 95\% CI 0.56-1.33). Additionally, there was no difference in the risk of cardiotoxicity comparing those with or without anthracycline treatment (HR $1.27,95 \%$ CI 0.88 to 
1.84). In conclusion, the cardiotoxic risks of taxanes are low.

\section{Targeted Therapy}

\section{Endocrine therapy}

Endocrine therapy is a corner stone in the treatment of hormone receptor positive breast cancer subtypes (ER/PR positive). In the adjuvant setting, endocrine therapy is given to selected patients for 5 to 10 years. Estrogen has indirect and direct effects on the cardiovascular system through the serum lipoprotein metabolism, coagulation, and fibrinolytic systems, and the antioxidative capacity. There have been concerns that estrogen inhibition in breast cancer treatment might mimic the postmenopausal effect on the cardiovascular system resulting in worsened cardiovascular outcome [21]. In breast cancer patients, antihormonal therapy is based on two major strategies, ER-modulators or inhibition of estrogen synthesis. Modulation of the ER is performed with selective ER modulators as tamoxifen, or estrogen receptor down-regulators as fulvestrant. The last two decades have been dominated by the introduction of aromatase inhibitors for postmenopausal women, inhibiting estrogen synthesis, i.e., with anastrozole, letrozole, and exemestane. Tamoxifen has shown to exert positive effects on the blood lipid profiles [22] and is believed to have a favorable cardiovascular effect [23], but its thromboembolic effect needs to be taken into consideration [24]. Aromatase inhibitors have been compared with placebo in several trials and do not seem to increase the risk for cardiovascular events in a significant manner [22]. However, these studies are not powered for cardiac events and frequently do not report specific cardiovascular adverse events. Aromatase inhibitors, when compared with tamoxifen, show an increased risk of arterial events as myocardial infarction and angina, which must be interpreted in the context of the protective effect of tamoxifen $[22,25]$. Tamoxifen is primarily given to premenopausal women, while aromatase inhibitors of the third generation, i.e., anastrozole, letrozole and exemestane, are used in postmenopausal women.

Mammalian target of rapamycin (mTOR) inhibitors may be used in combination with exemestane in selected postmenopausal breast cancer patients with metastatic disease and have shown improved progression free survival compared with exemestane alone [26, 27]. mTORs can exhibit an indirect effect on cardiovascular health by metabolic changes, i.e., glucose and lipid metabolism, but are generally well tolerated [28]. Overall, the cardiovascular tolerability of endocrine therapy is high.

\section{HER2 Targeted Drugs}

Approximately $15 \%$ of breast cancers are HER 2 positive and are associated with aggressive behavior of the tumor [29].
Trastuzumab, a humanized monoclonal antibody against the extracellular domain of HER2, is in breast cancer often used in combination with chemotherapy or endocrine treatment [30, 31]. Pertuzumab is a recombinant humanized monoclonal antibody that binds to the extracellular domain of HER2 and functions as a dimerization inhibitor. As pertuzumab and trastuzumab binds to different domains of the HER2, a combination therapy is more beneficial than trastuzumab alone [32].

The HER2 receptor (also known as ERBB2 receptor) is expressed on cardiomyocytes, where it is involved in intracellular signaling pathways controlling apoptosis, cell regeneration, and to some extent the contractile function [33]. Blocking HER2 by trastuzumab may cause left ventricular dysfunction [32], but the complete mechanism of trastuzumab-induced cardiotoxicity is still not fully understood. In the clinical setting trastuzumab-induced myocardial dysfunction is potentiated by prior treatment with anthracyclines, which is also supported by preclinical studies [34-37]. Other risk factors include age $>65$ years, high body mass index $>30 \mathrm{~kg} / \mathrm{m}^{2}$, hypertension, and lower LVEF at baseline, while there are still conflicting data on other cardiovascular risk factors as diabetes, coronary artery disease, valvular dysfunction, and left ventricular hypertrophy [34, 38]. Trastuzumab-induced left ventricular dysfunction is to some extent reversible when paused or discontinued [10, 39-41]. Other studies have shown a persistent reduction in the left ventricular function after trastuzumab cessation [42-44]. In a study with 251 HER2 positive breast cancer patients, 42 patients had a LVEF drop of $>10 \%$ unit to a value below $50 \%$, and $40 \%$ of these patients did not regain full cardiac function after enalapril and carvedilol treatment [42]. Whether this irreversibility is due to concomitant anthracycline toxicity is still unclear. The clinical presentation ranges from asymptomatic impaired left ventricular function to fulminant heart failure [32, 41]. In 2012 a Cochrane report based on randomized controlled trials of adjuvant therapy in breast cancer, the range of left ventricular dysfunction was between 7 and 34\%. The rate of clinical severe heart failure (New York Heart Association (NYHA) class III or IV) ranged between 0 and $4 \%$ [45]. The risk is higher in older patients. In a register study identifying more than 45,000 patients over 67 years and treated with adjuvant therapy for early breast cancer, the adjusted 3 year incidence of heart failure or left ventricular dysfunction was $31 \%$ for those treated with trastuzumab alone, $42 \%$ for those treated with trastuzumab and anthracycline, and $18 \%$ for patients without adjuvant therapy [46]. Dual therapy with trastuzumab and pertuzumab does not increase the risk of cardiotoxicity compared with trastuzumab alone [47, 48].

The tyrosine kinase inhibitors lapatinib and tucatinib are approved for the treatment of HER2-positive, trastuzumabresistant metastatic breast cancer $[49,50]$. Neratinib is in some countries approved for extended adjuvant treatment [51]. In clinical trials the risk of cardiotoxicity of these drugs are lower 
than for trastuzumab; however, these trials included a low risk cohort $[50,52,53]$.

In conclusion, anti-HER2 therapy with trastuzumab is associated with significant risk of developing cardiotoxicity. There is no evidence of increased risk of cardiotoxicity when pertuzumab is added to trastuzumab.

\section{CDK 4/6 Inhibitors}

Dysregulation of the cyclin-dependent kinases (CDK) 4/6-cyclin-D cell-cycle pathway induces a loss of mitotic regulation [54] and is associated with endocrine resistance in breast cancer [55]. Small-molecule CDK4/6-inhibitors as ribociclib, palbociclib, and abemaciclib have shown the ability to inhibit the growth of ER-positive breast cancer cells, act synergistically with antiestrogens, and reverse endocrine resistance [55-58]. CDK4/6 inhibitors with either aromatase inhibitors or antiestrogens are currently recommended as first-line treatment option for metastatic ER-positive and HER2-negative breast cancers [49]. Regarding cardiac safety, a range of different cardiovascular toxicities has been reported. Moderate QT prolongation has been observed with ribociclib, and ECG monitoring is advised [57]. In addition, the risk of venous thromboembolic events is increased compared with endocrine treatment only [55, 57]. In cell models and rodents, the CD4 activation pathway is important in early cardiomyocyte remodeling after myocardial infarction [59]; however, the clinical significance of this is unclear.

\section{ICI}

The use of immune checkpoint inhibitors (ICIs) has vastly improved clinical outcomes in several cancer types during the last decade. However, in breast cancer patients the effect of current ICI regimens has been less beneficial [60]. Atetzolizumab and pembrolizumab have been approved for breast cancer treatment $[61,62]$. As ICIs improve the activity of cytotoxic T-lymphocytes through blocking a major inhibitory pathway, these drugs may also cause a number of immune-related adverse events [63]. A feared but rare ICIrelated adverse event is myocarditis, which may be associated with mortality rates up to $50 \%[64,65]$. Other rare ICImediated cardiovascular toxicities are pericarditis, heart block, acute coronary syndromes, Takotsubo syndrome, and during longer treatment non-inflammatory heart failure [66]. The use of ICI is expected to increase, and the cardiovascular complications will be an important issue for further surveillance.

\section{Radiation Therapy}

The aim of radiation therapy may be curative, adjuvant, or palliative, and most patients receive radiation as part of a multimodal treatment strategy including surgery, chemotherapy, and targeted therapies. For most patients the gains of improved survival and organ-preservation outweigh the risks of normal tissue effects as fibrosis, necrosis, and pain. Serious life-threatening late effects as heart disease and secondary cancers are uncommon. Radiation-associated heart disease usually materializes more than a decade after treatment [67]. With improved cancer survival, more patients are at risk of suffering from radiation-associated heart disease.

Both the micro- and the macro- coronary circulation and the left sided cardiac valves can be affected. In breast cancer patients a dose-response relationship between radiation dose to the heart and risk of major coronary events or cardiac death was established. A relative risk of major coronary events or heart death increased by $7.4 \%$ per 1 Gy increases in mean heart dose. Importantly, there was no lower safe dose [68]. Pre-existing vascular disease may increase the risk of cardiac complications [69]. Radiation-associated heart disease has shown to be more lethal compared with heart disease not related to radiation [70]. Rarely, the radiation effect on the heart may occur acutely or subacutely (pericarditis and/or myocarditis), but usually, the effect occurs within 10 years in women with pre-existing coronary disease and $10+$ years in lower risk individuals where healthy coronary vessels were exposed to radiation. Restrictive cardiomyopathy or constrictive pericarditis represents an advanced stage of damage due to fibrosis of the myocardium or pericardium with severe diastolic dysfunction and signs and symptoms of congestive heart failure $[67,71]$. In conclusion radiation-induced cardiac disease may present decades after treatment completion.

\section{Detection}

\section{Imaging}

Cardiac dysfunction secondary to cancer treatment can be defined as an absolute drop in LVEF of $\geq 10 \%$ to a value below $<50 \%$ [10]. Nuclear multigated acquisition (MUGA) and echocardiography are the most common modalities for serial LVEF measurements. A significant decline in LVEF might influence the continuation of cancer treatment; hence, LVEF measurements should be repeated after 2-3 weeks to confirm the decline [72]. The method of choice and vendor should preferably be consistent through the surveillance period and adapted to local availability and expertise (Table 1). LVEF comparison between different modalities is not advisable. If possible, MUGA should be avoided mainly due to radiation exposure [10]. Cardiac magnetic resonance imaging (CMR) is a reliable method for assessment of cardiac function and is the gold standard to measure volumes and EF. CMR adds value in differential tissue diagnostics; however, its unavailability and high cost precludes a more general use. As new cancer therapy-induced adverse effects arise, the role of CMR may become more prominent. ICImediated perimyocarditis might be missed by simple LVEF measurement; instead, it should be examined by CMR with 
T1 and T2 mapping to detect myocardial edema along with late gadolinium contrast enhancement of the inflamed pericardium and myocardium [73].

When using echocardiography, the most reliable measurement of LVEF is the transthoracic three-dimensional volumetric method. When not available, biplane Simpson method from two-dimensional echocardiography should be used. Two-dimensional GLS measured using speckle tracking is a more sensitive method than LVEF to evaluate early changes in left ventricular systolic function and should be used together with $\operatorname{LVEF}[10,12 \bullet, 72]$. It has been suggested that a GLS value of $<18 \%$ or a relative reduction of $15 \%$ from baseline is significant [12•,72]. However, these thresholds are based on small observational studies. It is unconfirmed if early change in GLS values will predict persistent decrease in LVEF or the development of systolic heart failure. Results from the SUCCOUR (Strain sUrveillance of Chemotherapy for improving Cardiovascular Outcomes) study, a randomized controlled international multicenter trial of GLS and EF in surveillance of cardiotoxicity in cancer patient, is highly awaited [74]. The target inclusion is 320 patients with a follow-up time of 3 years. The results will hopefully provide evidence for whether the use of GLS for surveillance for cardiotoxicity improves clinical outcomes.

Other sensitive methods to measure cardiotoxicity, as changes in right heart function, diastolic function, and changes unveiled during exercise, are in their infancy regarding to their reliability, usability and overall clinical relevance [12••, 72, 75, 76].

\section{Echocardiographic Follow-Up During Treatment with Anthracycline and Trastuzumab}

Routine cardiac surveillance under anthracycline and HER2-treament has been recommended by both the cardiology and oncology working groups $[10,12 \bullet \bullet$, $77 \bullet \bullet$. Expert consensus papers argue that baseline cardiac function should be assessed before adjuvant anthracycline treatment, especially as part of baseline risk assessment $[10,72,76,77 \bullet \bullet, 78]$.

\section{Anthracyclines}

A baseline cardiac evaluation is desirable in all patients scheduled to receive anthracycline chemotherapy using the new Heart Failure Association of the European Society of Cardiology in collaboration with the International Cardio-Oncology Society (HFA-ICOS) baseline risk stratification proforma [79••]. Based on the accumulated dose-dependent toxicity of anthracyclines, follow-up is recommended, especially when cumulative doxorubicin-doses exceeds $240 \mathrm{mg} / \mathrm{m}^{2}$ [10, 77••]. These strategies have not been validated to prevent long-term cardiac events, nor has long-term follow-up after completion of anthracycline-therapy.

\section{Trastuzumab}

For HER2-targeted therapies, a baseline measurement of cardiac function by LVEF and GLS is suggested using the new HFA-ICOS baseline risk stratification proforma [79••]. According to The Food and Drug Administration (FDA) approval, serial cardiac evaluation during trastuzumab treatment should be performed every 3rd month. This is based on clinical trial protocols. However, this 'one size fits all' approach is not logical clinically, and a more personalized monitoring schedule based upon baseline risk has been suggested [79••]. Even though frequent cardiac surveillance are recommended

Table 1 Recommended imaging for detection and follow-up of cardiotoxicity in patients treated for breast cancer

\begin{tabular}{|c|c|c|c|c|c|}
\hline $\begin{array}{l}\text { Imaging } \\
\text { modality }\end{array}$ & Method & $\begin{array}{l}\text { Normal } \\
\text { range }\end{array}$ & $\begin{array}{l}\text { Detection of } \\
\text { cardiotoxcity }\end{array}$ & Pro/con & Recommendation \\
\hline Echo, 2D & $\begin{array}{l}\text { LVEF, biplane } \\
\text { Simpson }\end{array}$ & $>53 \%$ & $\begin{array}{l}\geq 10 \% \text { absolute } \\
\quad \text { change to a value } \\
\quad<50 \%\end{array}$ & $\begin{array}{l}\text { Widely accessible and used, but relatively } \\
\text { high variability }\end{array}$ & $\begin{array}{l}\text { Recommended in combination } \\
\text { with GLS and biomarkers }\end{array}$ \\
\hline $\begin{array}{l}\text { Echo, speckle } \\
\text { tracking }\end{array}$ & GLS & $>18 \%$ & $\begin{array}{l}\text { value }<18 \% \text { or }>15 \% \\
\text { relative reduction } \\
\text { from baseline }\end{array}$ & $\begin{array}{l}\text { High reliability and validity, sensitive for early } \\
\text { detection, especially in combination with } \\
\text { biomarkers. }\end{array}$ & $\begin{array}{l}\text { Recommended in combination } \\
\text { with } 2 \mathrm{D} \text { echo and biomarkers }\end{array}$ \\
\hline Echo, 3D & LVEF, 3D & $>55 \%$ & $\begin{array}{l}\geq 10 \% \text { absolute } \\
\quad \text { change to a value } \\
\quad<50 \%\end{array}$ & $\begin{array}{l}\text { High reliability, not so widely used, more } \\
\text { complicated than } 2 \mathrm{D}\end{array}$ & Recommended if available \\
\hline CMR & LVEF & $>55 \%$ & $\begin{array}{l}\geq 10 \% \text { absolute } \\
\quad \text { change to a value } \\
\quad<50 \%\end{array}$ & $\begin{array}{l}\text { Reliable method, low availability, add tissue } \\
\text { information when needed }\end{array}$ & $\begin{array}{l}\text { Recommended when tissue } \\
\text { information is necessary (i.e., } \\
\text { myocarditis) }\end{array}$ \\
\hline
\end{tabular}

$C M R$ cardiac magnetic resonance imaging, Echo echocardiography, GLS global longitudinal strain, $L V E F$ left ventricular ejection fraction 
[12••], studies on the optimal frequency of cardiac monitoring during trastuzumab treatment are lacking [80].

\section{Biomarkers}

Serum cardiac biomarkers indicating cardiac injury or dysfunction can be considered incorporated to the surveillance of patient at risk of cardiotoxicity. Even though a wide selection of different biomarkers has been studied, B-type natriuretic peptides (BNP) and cardiac troponins have been adopted to clinical practice. The value of BNP to detect cancer treatment related cardiotoxicity is limited by other variables that influence their levels including hemodynamic changes from stress, hypertension, infusions, vomiting, diarrhea, etc. A markedly elevated level of BNP has a high sensitivity for heart failure, while the specificity is variable [81]. It is unclear whether a rise in BNP precedes a decline in LVEF during breast cancer treatment [82]. Elevated levels of BNP during radiation therapy has been demonstrated, but the significance of this is unclear [83, 84]. While BNP is a marker of cardiac dysfunction, cardiac troponins are markers of myocardial injury. A rise in cardiac troponin I during high-dose anthracycline treatment has been a strong predictor of later LVEF decline and clinical events [85]. However, it is unclear if a rise in high sensitivity troponin precedes a decline in LVEF in contemporary breast cancer treatment [82]. Recently, the International CardioOncology Society-one trial (ICOSONE) evaluated the hypothesis of biomarker-guided cardioprotection versus primary prevention in 273 cancer patients scheduled to receive anthracycline chemotherapy, including breast cancer patients [86]. The results showed that the incidence of new troponin elevation was 23-26\% in both arms. Enalapril pre-treatment in primary prevention did not prevent the troponin elevation, but it did reduce maladaptive remodeling and LV dysfunction in the very few individuals who sustained a cardiac injury and troponin rise. This trial was limited by relatively small numbers, variability of the troponin assays used in the recruitment hospitals, a relatively low risk cohort, and $14 \%$ of patients in the primary prevention arm stopping enalapril due to side effects.

Previous guidelines recommend monitoring troponin in high-risk patients to guide the decisiveness of cardioprotective management $[10,77 \bullet \bullet]$. The most recent position statement from the HFA-ICOS recommends measurement at baseline pre-treatment to contribute to risk assessment [79••].

An increase in cardiac troponin is one of the diagnostic criteria for ICI-myocarditis [87]. Ideally, a cardiac troponin should be measured at baseline pre-treatment to place any future values in context. The benefit of routine cardiac troponin monitoring in all cancer patients receiving ICI without any cardiac symptoms is unclear.

\section{Preventive and Management Strategies}

\section{Risk Factor Assessment}

A pre-treatment cardiovascular risk assessment should be made for all patients using the new HFA-ICOS baseline risk stratification proformas to minimize the additional risk for developing cardiovascular disease during cancer treatment, i.e., evaluating baseline cardiac function, biomarkers, prior cardiovascular disease, and cardiovascular risk factors including glucose levels, lipid status, and checking blood pressure [79・•]. These factors should be treated according to guidelines (Fig. 1). Beta blocker and/or an angiotensin antagonist should be considered for the treatment of hypertension as these drugs have shown additional cardioprotective effect during anthracyclines and/or trastuzumab treatment [88-94].

Patients with established cardiovascular disease should be on optimal treatment, and a recent echocardiogram is essential to verify their cardiac function. A pre-existing diagnosis of heart failure does not necessarily exclude treatment with anthracyclines or trastuzumab but identifies high-risk patients. A careful cardiac evaluation should determine the reason for the heart failure, i.e., heart failure with reduced (HFrEF) or preserved ejection fraction (HFpEF) or reversible causes, i.e., arrhythmia, coronary disease with ischemia, or Takotsubo syndrome. Smaller studies are indicating that even those with reduced or decline in LVEF may tolerate HER2-targeted therapy under cardioprotective treatment and close cardiac monitoring [41, 95•, 96•]; however, larger studies are awaited to confirm these findings.

\section{Management of Left Ventricular Dysfunction}

In standard heart failure care and prevention there is strong evidence for symptom relief and mortality benefits for the treatment of HFrEF (LVEF < 40\%) [97]. Breast cancer patients presenting with $\mathrm{HFrEF}$ should receive standard heart failure care according to guidelines. This includes both pharmacological treatment as angiotensin antagonists, beta blockers, mineral corticoid receptor antagonists (MRA), sacubitril and ivabradine, and device treatment [81]. One known side effect of MRA spironolactone is gynecomastia due to peripheral conversion of testosterone to estradiol. Several studies have studied the use of spironolactone, but there has been no evidence of increased risk for breast cancer [98]. There is no proven treatment for HFpEF (LVEF > 50\%) and heart failure with midrange ejection fraction (HFmrEF) (LVEF between 40 and 50\%). Although the evidence is not strong, new studies support continuation of Trastuzumab if 


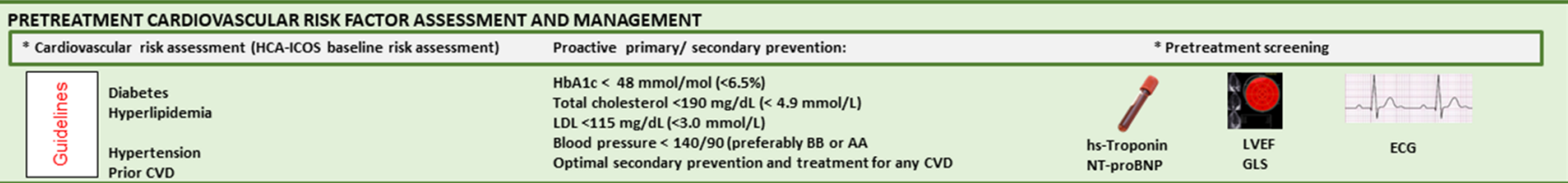

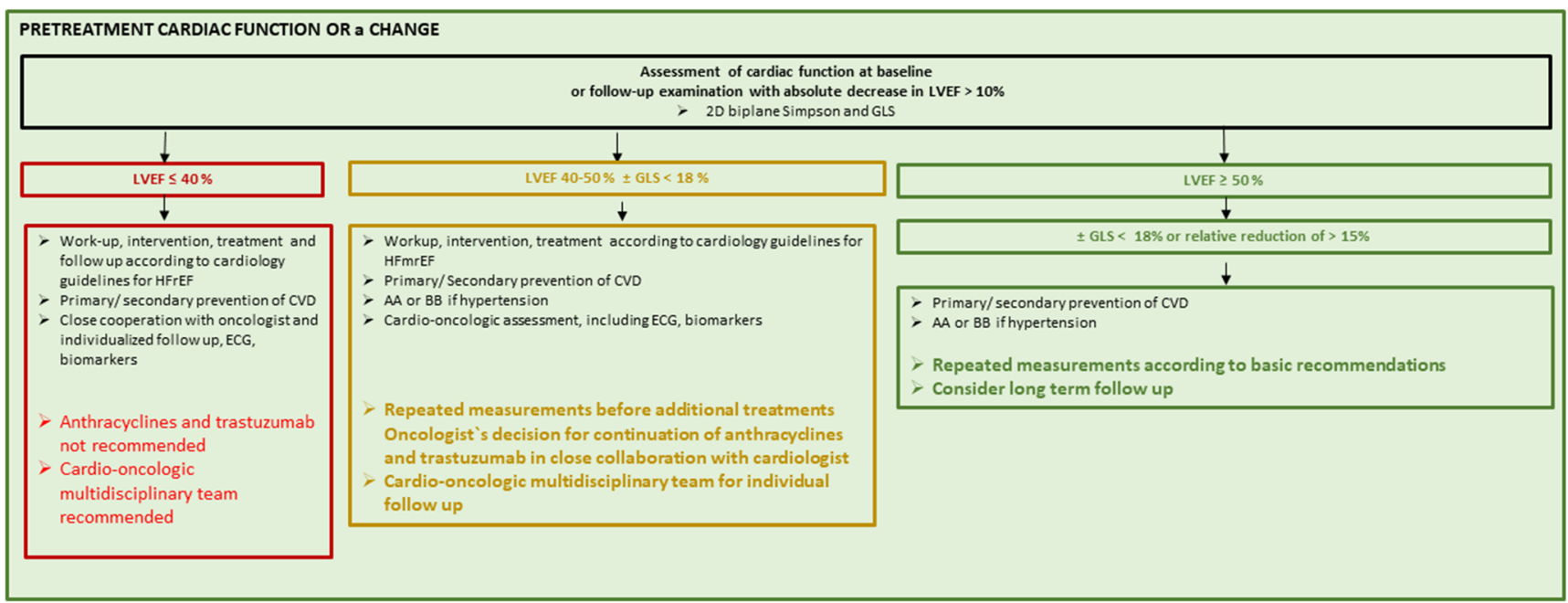

Fig. 1 Detection and management of heart failure in patients receiving treatment for breast cancer. AA angiotensin antagonist, BB beta blocker, CVD cardiovascular disease, ECG electrocardiographic, GLS global longitudinal strain, HCA-ICOS Heart Failure Association of the European Society of Cardiology in collaboration HFmEF heart failure

LVEF is $>40 \%$ in asymptomatic patients [95•, 96 ]. If LVEF drops $\geq 10 \%$ to a value $<50 \%$, treatment with angiotensin antagonists and beta blockers are recommended $[10,77 \bullet \bullet$, $95 \cdot, 96 \cdot$. Some early preventive studies during trastuzumab are more consistent with the protective effect of angiotensin antagonists than beta blockers [88-90, 92]. Data are lacking of when early cardioprotective treatment should be commenced, it should be evaluated based on change in cardiac function, elevated/rise in troponin and BNP, and cardiovascular comorbidities. Interestingly studies show that the use of beta blockers may attenuate the rise in cardiac troponin during anthracycline treatment, in none of these studies did beta blocker treatment effect the LVEF [90, 99]. The true meaning of mitigating the rise of troponin is unclear as long-term follow-up data is missing.

Dexrazoxane is well documented as a cardioprotective drug during anthracycline treatment [100]. Its use has been subdued because of concerns that it may either reduce the efficacy of anthracycline treatment against the primary malignancy or increase the risk of second malignancies. However, Cochrane Database Systemic Review analyses have shown no long-term safety concerns [100].

\section{Endocrine Treatment}

Compared with placebo neither tamoxifen nor aromatase inhibitors increase the risk of myocardial infarction. There are with midrange ejection fraction, HFrEF heart failure with reduced ejection fraction, Hs-Troponin high sensitive troponine with the International Cardio-Oncology Society, LDL low dense lipoprotein, LVEF left ventricular ejection fraction, NT-proBNP B-type natriuretic peptides

no studies comparing discontinuation-continuation of endocrine therapy if a cardiovascular event occurs.

\section{Radiation Therapy}

There is no evidence of cardioprotective treatment during radiation therapy. Complications should be treated according to general recommendations. However, it is important to keep in mind that irrradiation may also damage the microvasculature contributing to heart failure and that irrradiation often effects the proximal parts of the coronary arteries causing fibrotic lesions that are difficult to treat. Whether percutaneous coronary intervention (PCI) on these lesions is related to more complications is unclear $[101,102]$.

\section{Immune Checkpoint Inhibitors}

In patients with ICI-myocarditis early treatment with highdose intravenous methylprednisolone of $500-1000 \mathrm{mg}$ i.v. for 3 days is recommended. If improvement is seen, a switch to oral prednisolone of $0.5-1 \mathrm{mg} / \mathrm{kg}$ with a weekly weaning schedule can be made [66, 103•]. Delaying high-dose steroids in cases of ICI-myocarditis increases the risk of major adverse cardiac events including mortality [103•]. If the myocarditis does not settle with high-dose methylprednisolone, second line immunosuppressive drugs should be considered including mycophenolate mofetil and possibly abatacept [104]. 
Plasmapheresis can also be considered [105••, 106]. In asymptomatic cases where elevated troponin levels are detected by screening, oral prednisolone of $2 \mathrm{mg} / \mathrm{kg} /$ day may be considered $[77 \bullet \bullet, 105 \bullet \cdot$.

\section{Conclusion}

Modern breast cancer treatment is effective and has improved long-term survival. However, the long-term cardiovascular mortality and morbidity in these patients have also risen. The risk of left ventricular dysfunction and heart failure is substantially increased following treatment with anthracycline and trastuzumab. Chest wall radiation accelerates the atherosclerotic process and induces fibrosis. Immune checkpoint inhibitors are associated with fatal myocarditis. CDK4/6 inhibitors, cyclophosphamide, taxanes, tyrosine kinase inhibitors, and endocrine therapy have a lower-risk profile for cardiotoxicity.

Treatment of cardiovascular risk factors is recommended to avoid cardiotoxicity. Echocardiography and measuring circulating cardiovascular biomarkers such as troponins and BNP are useful as baseline markers and in evaluating the deleterious effect cancer treatment may have on the heart. The frequency of surveillance is based on expert consensus rather than randomized trials. HFA Cardio-Oncology Study Group has recently suggested a structured pathways for baseline risk assessment and surveillance. Beta blockers and angiotensin antagonists have shown promising results to prevent cardiotoxicity during anthracycline and trastuzumab treatment. Cardiopreventive techniques should be used in breast radiotherapy to minimize the cardiac radiation dose. If ICIinduced myocarditis is suspected, early intervention with high dose methyl-prednisolone has shown promising results.

Further clinical trials focusing on early detection, cardioprevention, and management are needed to elucidate the best strategies to prevent short- and long term development of left ventricular dysfunction and heart failure and thus promoting overall survival and quality of life.

Funding Open Access funding provided by University of Oslo (incl Oslo University Hospital).

\section{Compliance with Ethical Standards}

Conflict of Interest ARL has received speaker, advisory board or consultancy fees, and/or research grants from Pfizer, Novartis, Servier, Amgen, Takeda, Roche, Janssens-Cilag Ltd., Clinigen Group, Eli Lily, Eisai, Bristol Myers Squibb, Ferring Pharmaceuticals, Boehringer Ingelheim, Myocardial Solutions, Brainstorm Inc., iOWNA, and Heartfelt Technologies. All the author co-authors have no disclosures. GG has received speaker honoraria from Novartis, AstraZeneca, and Bristol-Myers Squibb. JG has received speaker, advisory board, and consultancy fees from Novartis, AstraZeneca, Pfizer, MSD, BMS, Lilly, and Pierre Fabre. TO reports research support via Akershus University
Hospital from Abbott Diagnostics, Novartis, Roche Diagnostics, Singulex, and SomaLogic and honoraria from Siemens Healthineers, Abbott Diagnostics, Roche Diagnostics, and CardiNor. AMB, ST, TS, $\mathrm{TJH}, \mathrm{KMN}, \mathrm{EH}$, and BVO have no disclosures.

Human and Animal Rights and Informed Consent This article does not contain any studies with human or animal subjects performed by any of the authors.

Abbreviations $B N P$, B-type natriuretic peptides; $C D K$, Cyclin-dependent kinases; $C M R$, Cardiovascular magnetic resonance imaging; $D N A$, Deoxyribonucleic acid; $E C G$, Electrocardiogram; $E R$, Estrogen receptor; $G L S$, Global longitudinal strain; HER (ERBB), Epidermal growth factor receptor (Receptor tyrosine-protein kinase); HFA-ICOS, Heart Failure Association of the European Society of Cardiology in collaboration with the International Cardio-Oncology Society; $L V E F$, Left ventricular ejection fraction; $m T O R$, Mammalian target of rapamycin; MUGA, Nuclear multigated acquisition; $P R$, Progesterone receptor

Open Access This article is licensed under a Creative Commons Attribution 4.0 International License, which permits use, sharing, adaptation, distribution and reproduction in any medium or format, as long as you give appropriate credit to the original author(s) and the source, provide a link to the Creative Commons licence, and indicate if changes were made. The images or other third party material in this article are included in the article's Creative Commons licence, unless indicated otherwise in a credit line to the material. If material is not included in the article's Creative Commons licence and your intended use is not permitted by statutory regulation or exceeds the permitted use, you will need to obtain permission directly from the copyright holder. To view a copy of this licence, visit http://creativecommons.org/licenses/by/4.0/.

\section{References}

Papers of particular interest, published recently, have been highlighted as:

- Of importance

•• Of major importance

1. https://gco.iarc.fr/today. Accessed 30 June 20.

2. Abdel-Qadir H, Austin PC, Lee DS, Amir E, Tu JV, Thavendiranathan $\mathrm{P}$, et al. A population-based study of cardiovascular mortality following early-stage breast Cancer. JAMA Cardiol. 2017;2(1):88-93.

3. Yeh ET, Chang HM. Oncocardiology-past, present, and future: a review. JAMA Cardiol. 2016;1(9):1066-72.

4. Vejpongsa P, Yeh ET. Prevention of anthracycline-induced cardiotoxicity: challenges and opportunities. J Am Coll Cardiol. 2014;64(9):938-45.

5. Zhang S, Liu X, Bawa-Khalfe T, Lu LS, Lyu YL, Liu LF, et al. Identification of the molecular basis of doxorubicin-induced cardiotoxicity. Nat Med. 2012;18(11):1639-42.

6. Herrmann J. Adverse cardiac effects of cancer therapies: cardiotoxicity and arrhythmia. Nat Rev Cardiol. 2020;17:474502.

7. Swain SM, Whaley FS, Ewer MS. Congestive heart failure in patients treated with doxorubicin: a retrospective analysis of three trials. Cancer. 2003;97(11):2869-79. 
8. Charbonnel C, Convers-Domart R, Rigaudeau S, Taksin AL, Baron N, Lambert J, et al. Assessment of global longitudinal strain at low-dose anthracycline-based chemotherapy, for the prediction of subsequent cardiotoxicity. Eur Heart J Cardiovasc Imaging. 2017;18(4):392-401.

9. Ferrans VJ. Overview of cardiac pathology in relation to anthracycline cardiotoxicity. Cancer Treat Rep. 1978;62(6):95561.

10. Zamorano JL, Lancellotti P, Rodriguez Munoz D, Aboyans V, Asteggiano R, Galderisi M, et al. 2016 ESC Position Paper on cancer treatments and cardiovascular toxicity developed under the auspices of the ESC Committee for Practice Guidelines: the task force for cancer treatments and cardiovascular toxicity of the European Society of Cardiology (ESC). Eur Heart J. 2016;37(36): 2768-801.

11. Cardinale D, Iacopo F, Cipolla CM. Cardiotoxicity of anthracyclines. Front Cardiovasc Med. 2020;7:26.

12.• Celutkiene J, Pudil R, Lopez-Fernandez T, Grapsa J, Nihoyannopoulos P, Bergler-Klein J, et al. The role of cardiovascular imaging in cancer patients receiving cardiotoxic therapies: a Position statement on behalf of the Heart Failure Association (HFA), the European Association of Cardiovascular Imaging (EACVI) and the Cardio-Oncology Council of the European Society of Cardiology (ESC). Eur J Heart Fail. 2020 Jul 4. https://doi.org/10.1002/ejhf.1957. This paper provides a thorough insight of cardiovascular imgaing in cancer patietns receiving cardiotoxic therapies.

13. Iqubal A, Iqubal MK, Sharma S, Ansari MA, Najmi AK, Ali SM, et al. Molecular mechanism involved in cyclophosphamideinduced cardiotoxicity: old drug with a new vision. Life Sci. 2019;218:112-31.

14. Jackson GH, Davies FE, Pawlyn C, Cairns DA, Striha A, Collett $\mathrm{C}$, et al. Response-adapted intensification with cyclophosphamide, bortezomib, and dexamethasone versus no intensification in patients with newly diagnosed multiple myeloma (myeloma XI): a multicentre, open-label, randomised, phase 3 trial. Lancet Haematol. 2019;6(12):e616-e29.

15. Martel S, Maurer C, Lambertini M, Ponde N, De Azambuja E. Breast cancer treatment-induced cardiotoxicity. Expert Opin Drug Saf. 2017;16(9):1021-38.

16. Martin M, Pienkowski T, Mackey J, Pawlicki M, Guastalla JP, Weaver C, et al. Adjuvant docetaxel for node-positive breast cancer. N Engl J Med. 2005;352(22):2302-13.

17. Biganzoli L, Cufer T, Bruning P, Coleman RE, Duchateau L, Rapoport B, et al. Doxorubicin-paclitaxel: a safe regimen in terms of cardiac toxicity in metastatic breast carcinoma patients. Results from a European Organization for Research and Treatment of Cancer multicenter trial. Cancer. 2003;97(1):40-5.

18. Marty M, Cognetti F, Maraninchi D, Snyder R, Mauriac L, Tubiana-Hulin M, et al. Randomized phase II trial of the efficacy and safety of trastuzumab combined with docetaxel in patients with human epidermal growth factor receptor 2-positive metastatic breast cancer administered as first-line treatment: the M77001 study group. J Clin Oncol. 2005;23(19):4265-74.

19. Madeddu C, Deidda M, Piras A, Cadeddu C, Demurtas L, Puzzoni $\mathrm{M}$, et al. Pathophysiology of cardiotoxicity induced by nonanthracycline chemotherapy. J Cardiovasc Med (Hagerstown). 2016;17 Suppl 1 Special issue on Cardiotoxicity from Antiblastic Drugs and Cardioprotection:e12-e8.

20. Willson ML, Burke L, Ferguson T, Ghersi D, Nowak AK, Wilcken N. Taxanes for adjuvant treatment of early breast cancer. Cochrane Database Syst Rev. 2019;9:CD004421.

21. Menazza S, Murphy E. The expanding complexity of estrogen receptor signaling in the cardiovascular system. Circ Res. 2016;118(6):994-1007.
22. Khosrow-Khavar F, Filion KB, Al-Qurashi S, Torabi N, Bouganim N, Suissa S, et al. Cardiotoxicity of aromatase inhibitors and tamoxifen in postmenopausal women with breast cancer: a systematic review and meta-analysis of randomized controlled trials. Ann Oncol. 2017;28(3):487-96.

23. Grainger DJ, Schofield PM. Tamoxifen for the prevention of myocardial infarction in humans: preclinical and early clinical evidence. Circulation. 2005;112(19):3018-24.

24. Decensi A, Maisonneuve P, Rotmensz N, Bettega D, Costa A, Sacchini V, et al. Effect of tamoxifen on venous thromboembolic events in a breast cancer prevention trial. Circulation. 2005;111(5):650-6.

25. Haque R, Shi J, Schottinger JE, Chung J, Avila C, Amundsen B, et al. Cardiovascular disease after aromatase inhibitor use. JAMA Oncol. 2016;2(12):1590-7.

26. Jerusalem G, Mariani G, Ciruelos EM, Martin M, Tjan-Heijnen VC, Neven P, et al. Safety of everolimus plus exemestane in patients with hormone-receptor-positive, HER2-negative locally advanced or metastatic breast cancer progressing on prior nonsteroidal aromatase inhibitors: primary results of a phase IIIb, open-label, single-arm, expanded-access multicenter trial (BALLET). Ann Oncol. 2016;27(9):1719-25.

27. Piccart M, Hortobagyi GN, Campone M, Pritchard KI, Lebrun F, Ito $\mathrm{Y}$, et al. Everolimus plus exemestane for hormone-receptorpositive, human epidermal growth factor receptor-2-negative advanced breast cancer: overall survival results from BOLERO2dagger. Ann Oncol. 2014;25(12):2357-62.

28. Karvelas G, Roumpi A, Komporozos C, Syrigos K. Everolimus as cancer therapy: Cardiotoxic or an unexpected antiatherogenic agent? A narrative review. Hell J Cardiol. 2018;59(4):196-200.

29. Varga Z, Noske A, Ramach C, Padberg B, Moch H. Assessment of HER2 status in breast cancer: overall positivity rate and accuracy by fluorescence in situ hybridization and immunohistochemistry in a single institution over 12 years: a quality control study. BMC Cancer. 2013;13:615.

30. Slamon DJ, Clark GM, Wong SG, Levin WJ, Ullrich A, McGuire WL. Human breast cancer: correlation of relapse and survival with amplification of the HER-2/neu oncogene. Science. 1987;235(4785):177-82.

31. Vogel CL, Cobleigh MA, Tripathy D, Gutheil JC, Harris LN, Fehrenbacher L, et al. Efficacy and safety of trastuzumab as a single agent in first-line treatment of HER2-overexpressing metastatic breast cancer. J Clin Oncol. 2002;20(3):719-26.

32. Ishii K, Morii N, Yamashiro H. Pertuzumab in the treatment of HER2-positive breast cancer: an evidence-based review of its safety, efficacy, and place in therapy. Core Evid. 2019;14:51-70.

33. Rochette L, Guenancia C, Gudjoncik A, Hachet O, Zeller M, Cottin Y, et al. Anthracyclines/trastuzumab: new aspects of cardiotoxicity and molecular mechanisms. Trends Pharmacol Sci. 2015;36(6):326-48.

34. Moilanen T, Jokimaki A, Tenhunen O, Koivunen JP. Trastuzumab-induced cardiotoxicity and its risk factors in realworld setting of breast cancer patients. J Cancer Res Clin Oncol. 2018;144(8):1613-21.

35. Florido R, Smith KL, Cuomo KK, Russell SD. Cardiotoxicity From Human Epidermal Growth Factor Receptor-2 (HER2) Targeted Therapies. Am Heart Assoc. 2017 Sep 22;6(9): e006915. https://doi.org/10.1161/JAHA.117.006915.

36. Halyard MY, Pisansky TM, Dueck AC, Suman V, Pierce L, Solin $\mathrm{L}$, et al. Radiotherapy and adjuvant trastuzumab in operable breast cancer: tolerability and adverse event data from the NCCTG Phase III Trial N9831. J Clin Oncol. 2009;27(16):2638-44.

37. Bowles EJ, Wellman R, Feigelson HS, Onitilo AA, Freedman AN, Delate T, et al. Risk of heart failure in breast cancer patients after anthracycline and trastuzumab treatment: a retrospective cohort study. J Natl Cancer Inst. 2012;104(17):1293-305. 
38. Tang GH, Acuna SA, Sevick L, Yan AT, Brezden-Masley C. Incidence and identification of risk factors for trastuzumabinduced cardiotoxicity in breast cancer patients: an audit of a single "real-world" setting. Med Oncol. 2017;34(9):154.

39. Hudis CA. Trastuzumab-mechanism of action and use in clinical practice. N Engl J Med. 2007;357(1):39-51.

40. Onitilo AA, Engel JM, Stankowski RV. Cardiovascular toxicity associated with adjuvant trastuzumab therapy: prevalence, patient characteristics, and risk factors. Ther Adv Drug Saf. 2014;5(4): 154-66.

41. Hussain Y, Drill E, Dang CT, Liu JE, Steingart RM, Yu AF. Cardiac outcomes of trastuzumab therapy in patients with HER2-positive breast cancer and reduced left ventricular ejection fraction. Breast Cancer Res Treat. 2019;175(1):239-46.

42. Cardinale D, Colombo A, Torrisi R, Sandri MT, Civelli M, Salvatici M, et al. Trastuzumab-induced cardiotoxicity: clinical and prognostic implications of troponin I evaluation. J Clin Oncol. 2010;28(25):3910-6.

43. Romond EH, Jeong JH, Rastogi P, Swain SM, Geyer CE Jr, Ewer MS, et al. Seven-year follow-up assessment of cardiac function in NSABP B-31, a randomized trial comparing doxorubicin and cyclophosphamide followed by paclitaxel (ACP) with ACP plus trastuzumab as adjuvant therapy for patients with node-positive, human epidermal growth factor receptor 2-positive breast cancer. J Clin Oncol. 2012;30(31):3792-9.

44. Ohtani K, Ide T, Hiasa KI, Sakamoto I, Yamashita N, Kubo M, et al. Cardioprotective effect of renin-angiotensin inhibitors and beta-blockers in trastuzumab-related cardiotoxicity. Clin Res Cardiol. 2019;108(10):1128-39.

45. Moja L, Tagliabue L, Balduzzi S, Parmelli E, Pistotti V, Guarneri $\mathrm{V}$, et al. Trastuzumab containing regimens for early breast cancer. Cochrane Database Syst Rev. 2012;4:CD006243.

46. Chen J, Long JB, Hurria A, Owusu C, Steingart RM, Gross CP. Incidence of heart failure or cardiomyopathy after adjuvant trastuzumab therapy for breast cancer. J Am Coll Cardiol. 2012;60(24):2504-12.

47. Swain SM, Baselga J, Kim SB, Ro J, Semiglazov V, Campone M, et al. Pertuzumab, trastuzumab, and docetaxel in HER2-positive metastatic breast cancer. N Engl J Med. 2015;372(8):724-34.

48. Yu AF, Manrique C, Pun S, Liu JE, Mara E, Fleisher M, et al. Cardiac safety of paclitaxel plus trastuzumab and pertuzumab in patients with HER2-positive metastatic breast Cancer. Oncologist. 2016;21(4):418-24.

49. Cardoso F, Senkus E, Costa A, Papadopoulos E, Aapro M, Andre $\mathrm{F}$, et al. 4th ESO-ESMO International Consensus Guidelines for Advanced Breast Cancer (ABC 4)dagger. Ann Oncol. 2018;29(8): 1634-57.

50. Murthy RK, Loi S, Okines A, Paplomata E, Hamilton E, Hurvitz SA, et al. Tucatinib, trastuzumab, and capecitabine for HER2positive metastatic breast cancer. N Engl J Med. 2020;382(7): 597-609.

51. Martin M, Holmes FA, Ejlertsen B, Delaloge S, Moy B, Iwata H, et al. Neratinib after trastuzumab-based adjuvant therapy in HER2-positive breast cancer (ExteNET): 5-year analysis of a randomised, double-blind, placebo-controlled, phase 3 trial. Lancet Oncol. 2017;18(12):1688-700.

52. Saura C, Garcia-Saenz JA, Xu B, Harb W, Moroose R, Pluard T, et al. Safety and efficacy of neratinib in combination with capecitabine in patients with metastatic human epidermal growth factor receptor 2-positive breast cancer. J Clin Oncol. 2014;32(32): 3626-33.

53. Eiger D, Ponde NF, Agbor-Tarh D, Moreno-Aspitia A, Piccart M, Hilbers FS, et al. Long-term cardiac outcomes of patients with HER2-positive breast cancer treated in the adjuvant lapatinib and/or trastuzumab treatment optimization trial. Br J Cancer. 2020;122(10):1453-60.
54. Malumbres M, Barbacid M. To cycle or not to cycle: a critical decision in cancer. Nat Rev Cancer. 2001;1(3):222-31.

55. Finn RS, Aleshin A, Slamon DJ. Targeting the cyclin-dependent kinases (CDK) 4/6 in estrogen receptor-positive breast cancers. Breast Cancer Res. 2016;18(1):17.

56. Finn RS, Dering J, Conklin D, Kalous O, Cohen DJ, Desai AJ, et al. PD 0332991, a selective cyclin D kinase 4/6 inhibitor, preferentially inhibits proliferation of luminal estrogen receptorpositive human breast cancer cell lines in vitro. Breast Cancer Res. 2009;11(5):R77.

57. Hortobagyi GN, Stemmer SM, Burris HA, Yap YS, Sonke GS, Paluch-Shimon S, et al. Updated results from MONALEESA-2, a phase III trial of first-line ribociclib plus letrozole versus placebo plus letrozole in hormone receptor-positive, HER2-negative advanced breast cancer. Ann Oncol. 2018;29(7):1541-7.

58. Goetz MP, Suman VJ, Reid JM, Northfelt DW, Mahr MA, Ralya AT, et al. First-in-human phase I study of the Tamoxifen metabolite Z-Endoxifen in women with endocrine-refractory metastatic breast Cancer. J Clin Oncol. 2017;35(30):3391-400.

59. Mohamed TMA, Ang YS, Radzinsky E, Zhou P, Huang Y, Elfenbein A, et al. Regulation of cell cycle to stimulate adult cardiomyocyte proliferation and cardiac regeneration. Cell. 2018;173(1):104-16 e12.

60. Emens LA. Breast Cancer immunotherapy: facts and hopes. Clin Cancer Res. 2018;24(3):511-20.

61. Schmid P, Rugo HS, Adams S, Schneeweiss A, Barrios CH, Iwata $\mathrm{H}$, et al. Atezolizumab plus nab-paclitaxel as first-line treatment for unresectable, locally advanced or metastatic triple-negative breast cancer (IMpassion130): updated efficacy results from a randomised, double-blind, placebo-controlled, phase 3 trial. Lancet Oncol. 2020;21(1):44-59.

62. Schmid P, Salgado R, Park YH, Munoz-Couselo E, Kim SB, Sohn $\mathrm{J}$, et al. Pembrolizumab plus chemotherapy as neoadjuvant treatment of high-risk, early-stage triple-negative breast cancer: results from the phase $1 \mathrm{~b}$ open-label, multicohort KEYNOTE-173 study. Ann Oncol. 2020;31(5):569-81.

63. Boutros C, Tarhini A, Routier E, Lambotte O, Ladurie FL, Carbonnel F, et al. Safety profiles of anti-CTLA-4 and anti-PD1 antibodies alone and in combination. Nat Rev Clin Oncol. 2016;13(8):473-86.

64. Mahmood SS, Fradley MG, Cohen JV, Nohria A, Reynolds KL, Heinzerling LM, et al. Myocarditis in patients treated with immune checkpoint inhibitors. J Am Coll Cardiol. 2018;71(16): 1755-64.

65. Salem JE, Manouchehri A, Moey M, Lebrun-Vignes B, Bastarache L, Pariente A, et al. Cardiovascular toxicities associated with immune checkpoint inhibitors: an observational, retrospective, pharmacovigilance study. Lancet Oncol. 2018;19(12): 1579-89.

66. Lyon AR, Yousaf N, Battisti NML, Moslehi J, Larkin J. Immune checkpoint inhibitors and cardiovascular toxicity. Lancet Oncol. 2018;19(9):e447-e58.

67. Nielsen KM, Offersen BV, Nielsen HM, Vaage-Nilsen M, Yusuf $\mathrm{SW}$. Short and long term radiation induced cardiovascular disease in patients with cancer. Clin Cardiol. 2017;40(4):255-61.

68. Darby SC, Ewertz M, McGale P, Bennet AM, Blom-Goldman U, Bronnum D, et al. Risk of ischemic heart disease in women after radiotherapy for breast cancer. N Engl J Med. 2013;368(11):98798.

69. Rehammar JC, Jensen MB, McGale P, Lorenzen EL, Taylor C, Darby SC, et al. Risk of heart disease in relation to radiotherapy and chemotherapy with anthracyclines among 19,464 breast cancer patients in Denmark, 1977-2005. Radiother Oncol. 2017;123(2):299-305. 
70. Boekel NB, Boekel LY, Buddeke J, Jacobse JN, Schaapveld M, Hooning MJ, et al. Prognosis of acute coronary syndromes after radiotherapy for breast cancer. Radiother Oncol. 2020;146:110-7.

71. Mulrooney DA, Yeazel MW, Kawashima T, Mertens AC, Mitby $\mathrm{P}$, Stovall M, et al. Cardiac outcomes in a cohort of adult survivors of childhood and adolescent cancer: retrospective analysis of the childhood cancer survivor study cohort. BMJ. 2009;339:b4606.

72. Plana JC, Galderisi M, Barac A, Ewer MS, Ky B, ScherrerCrosbie M, et al. Expert consensus for multimodality imaging evaluation of adult patients during and after cancer therapy: a report from the American Society of Echocardiography and the European Association of Cardiovascular Imaging. J Am Soc Echocardiogr. 2014;27(9):911-39.

73. Yu AF, Chan AT, Steingart RM. Cardiac magnetic resonance and cardio-oncology: does T2 signal the end of anthracycline cardiotoxicity? J Am Coll Cardiol. 2019;73(7):792-4.

74. Negishi T, Thavendiranathan P, Negishi K, Marwick TH. Investigators $\mathrm{S}$. rationale and design of the strain surveillance of chemotherapy for improving cardiovascular outcomes: the SUCCOUR trial. JACC Cardiovasc Imaging. 2018;11(8):1098105.

75. Saijo Y, Kusunose K, Yamada N, Yamada H, Nishio S, Hirata Y, et al. Sequential speckle tracking imaging to detect early stage of cancer therapeutics-related cardiac dysfunction in a patient with breast cancer. J Echocardiogr. 2020;18(2):134-5.

76. Eschenhagen T, Force T, Ewer MS, de Keulenaer GW, Suter TM, Anker SD, et al. Cardiovascular side effects of cancer therapies: a position statement from the Heart Failure Association of the European Society of cardiology. Eur J Heart Fail. 2011;13(1):110.

77.• Curigliano G, Lenihan D, Fradley M, Ganatra S, Barac A, Blaes A, et al. Management of cardiac disease in cancer patients throughout oncological treatment: ESMO consensus recommendations. Ann Oncol. 2020;31(2):171-90 This paper provides a thorough insight of cardiotoxicity and suggested treatment of the cardiotoxocity during cancer treatment.

78. Armenian SH, Lacchetti C, Lenihan D. Prevention and monitoring of cardiac dysfunction in survivors of adult cancers: American Society of Clinical Oncology clinical practice guideline summary. J Oncol Pract. 2017;13(4):270-5.

79.• Lyon AR, Dent S, Stanway S, Earl H, Brezden-Masley C, CohenSolal A, et al. Baseline cardiovascular risk assessment in cancer patients scheduled to receive cardiotoxic cancer therapies: a Position Statement and new risk assessment tools from the Cardio-Oncology Study Group of the Heart Failure Association of the European Society of Cardiology in collaboration with the International Cardio-Oncology Society. Eur J Heart Fail. 2020 May 28. https://doi.org/10.1002/ejhf.1920. This paper provides helpful risk assessement scores in cancer patients scheduled to receive cardiotoxic cancer therapies.

80. Bouwer NI, Jager A, Liesting C, Kofflard MJM, Brugts JJ, Kitzen $\mathrm{J}$, et al. Cardiac monitoring in HER2-positive patients on trastuzumab treatment: a review and implications for clinical practice. Breast. 2020;52:33-44.

81. Roberts E, Ludman AJ, Dworzynski K, Al-Mohammad A, Cowie MR, McMurray JJ, et al. The diagnostic accuracy of the natriuretic peptides in heart failure: systematic review and diagnostic metaanalysis in the acute care setting. BMJ. 2015;350:h910.

82. Tian S, Hirshfield KM, Jabbour SK, Toppmeyer D, Haffty BG, Khan AJ, et al. Serum biomarkers for the detection of cardiac toxicity after chemotherapy and radiation therapy in breast cancer patients. Front Oncol. 2014;4:277

83. Zhang C, Shi D, Yang P. BNP as a potential biomarker for cardiac damage of breast cancer after radiotherapy: a meta-analysis. Medicine (Baltimore). 2019;98(29):e16507.
84. Palumbo I, Palumbo B, Fravolini ML, Marcantonini M, Perrucci $\mathrm{E}$, Latini ME, et al. Brain natriuretic peptide as a cardiac marker of transient radiotherapy-related damage in left-sided breast cancer patients: a prospective study. Breast. 2016;25:45-50.

85. Cardinale D, Sandri MT, Colombo A, Colombo N, Boeri M, Lamantia G, et al. Prognostic value of troponin I in cardiac risk stratification of cancer patients undergoing high-dose chemotherapy. Circulation. 2004;109(22):2749-54.

86. Meessen J, Cardinale D, Ciceri F, Sandri MT, Civelli M, Bottazzi $\mathrm{B}$, et al. Circulating biomarkers and cardiac function over 3 years after chemotherapy with anthracyclines: the ICOS-ONE trial. ESC Heart Fail. 2020;7:1452-66.

87. Bonaca MP, Olenchock BA, Salem JE, Wiviott SD, Ederhy S, Cohen A, et al. Myocarditis in the setting of Cancer therapeutics: proposed case definitions for emerging clinical syndromes in cardio-oncology. Circulation. 2019;140(2):80-91.

88. Guglin M, Krischer J, Tamura R, Fink A, Bello-Matricaria L, McCaskill-Stevens W, et al. Randomized trial of Lisinopril versus carvedilol to prevent trastuzumab cardiotoxicity in patients with breast Cancer. J Am Coll Cardiol. 2019;73(22):2859-68.

89. Pituskin E, Mackey JR, Koshman S, Jassal D, Pitz M, Haykowsky MJ, et al. Multidisciplinary Approach to Novel Therapies in Cardio-Oncology Research (MANTICORE 101-Breast): a randomized trial for the prevention of trastuzumab-associated cardiotoxicity. J Clin Oncol. 2017;35(8):870-7.

90. Avila MS, Ayub-Ferreira SM, de Barros Wanderley MR Jr, das Dores Cruz F, Goncalves Brandao SM, Rigaud VOC, et al. Carvedilol for prevention of chemotherapy-related cardiotoxicity: the CECCY trial. J Am Coll Cardiol. 2018;71(20):2281-90.

91. Nabati M, Janbabai G, Baghyari S, Esmaili K, Yazdani J. Cardioprotective effects of carvedilol in inhibiting doxorubicininduced cardiotoxicity. J Cardiovasc Pharmacol. 2017;69(5):27985.

92. Gulati G, Heck SL, Ree AH, Hoffmann P, Schulz-Menger J, Fagerland MW, et al. Prevention of cardiac dysfunction during adjuvant breast cancer therapy (PRADA): a 2 × 2 factorial, randomized, placebo-controlled, double-blind clinical trial of candesartan and metoprolol. Eur Heart J. 2016;37(21):1671-80.

93. Kaya MG, Sarli B, Akpek M, Kaya EG, Yarlioglues M, Topsakal $\mathrm{R}$, et al. Evaluation of beta-blockers on left ventricular dyssynchrony and reverse remodeling in idiopathic dilated cardiomyopathy: a randomized trial of carvedilol and metoprolol. Cardiol J. 2014;21(4):434-41.

94. Tashakori Beheshti A, Mostafavi Toroghi H, Hosseini G, Zarifian A, Homaei Shandiz F, Fazlinezhad A. Carvedilol administration can prevent doxorubicin-induced cardiotoxicity: a double-blind randomized trial. Cardiology. 2016;134(1):47-53.

95. Lynce F, Barac A, Geng X, Dang C, Yu AF, Smith KL, et al. Prospective evaluation of the cardiac safety of HER2-targeted therapies in patients with HER2-positive breast cancer and compromised heart function: the SAFE-HEaRt study. Breast Cancer Res Treat. 2019;175(3):595-603 This study is insightful in showing safely continuation of trastuzumab with reduced LVEF.

96. Barron CC, Alhussein MM, Kaur U, Cosman TL, Tyagi NK, Brown M, et al. An evaluation of the safety of continuing trastuzumab despite overt left ventricular dysfunction. Curr Oncol. 2019;26(4):240-6 This study is insightful in showing safely continuation of trastuzumab with reduced LVEF.

97. Ponikowski P, Voors AA, Anker SD, Bueno H, Cleland JGF, Coats AJS, et al. 2016 ESC guidelines for the diagnosis and treatment of acute and chronic heart failure. Rev Esp Cardiol (Engl Ed). 2016;69(12):1167.

98. Rozner RN, Freites-Martinez A, Shapiro J, Geer EB, Goldfarb S, Lacouture ME. Safety of 5alpha-reductase inhibitors and 
spironolactone in breast cancer patients receiving endocrine therapies. Breast Cancer Res Treat. 2019;174(1):15-26.

99. Gulati G, Heck SL, Rosjo H, Ree AH, Hoffmann P, Hagve TA, et al. Neurohormonal Blockade and circulating cardiovascular biomarkers during anthracycline therapy in breast cancer patients: results from the PRADA (Prevention of Cardiac Dysfunction During Adjuvant Breast Cancer Therapy) Study. J Am Heart Assoc. 2017 Nov 8;6(11):e006513.

100. van Dalen EC, Caron HN, Dickinson HO, Kremer LC. Cardioprotective interventions for cancer patients receiving anthracyclines. Cochrane Database Syst Rev. 2011;6:CD003917.

101. Cuomo JR, Javaheri SP, Sharma GK, Kapoor D, Berman AE, Weintraub NL. How to prevent and manage radiation-induced coronary artery disease. Heart. 2018;104(20):1647-53.

102. Iliescu C, Grines CL, Herrmann J, Yang EH, Cilingiroglu M, Charitakis K, et al. SCAI expert consensus statement: evaluation, management, and special considerations of cardio-oncology patients in the cardiac catheterization laboratory (endorsed by the Cardiological Society of India, and Sociedad Latino Americana de Cardiologia Intervencionista). Catheter Cardiovasc Interv. 2016;87(5):895-9.

103. Zhang L, Zlotoff DA, Awadalla M, Mahmood SS, Nohria A, Hassan MZO, et al. Major Adverse Cardiovascular Events and the Timing and Dose of Corticosteroids in Immune Checkpoint Inhibitor-Associated Myocarditis. Circulation. 2020;141(24): 2031-4 This study is insightful in treatment of high dose methyprednisolone in ICI-induced myocarditis.

104. Salem JE, Allenbach Y, Vozy A, Brechot N, Johnson DB, Moslehi JJ, et al. Abatacept for severe immune checkpoint inhibitor-associated myocarditis. N Engl J Med. 2019;380(24): 2377-9.

105.• Hu JR, Florido R, Lipson EJ, Naidoo J, Ardehali R, Tocchetti CG, et al. Cardiovascular toxicities associated with immune checkpoint inhibitors. Cardiovasc Res. 2019;115(5):854-68 This paper is thorough in diagnosis and treatment of ICI-induced myocarditis.

106. Palaskas N, Lopez-Mattei J, Durand JB, Iliescu C, Deswal A. Immune checkpoint inhibitor myocarditis: pathophysiological characteristics, diagnosis, and treatment. J Am Heart Assoc. 2020;9(2): $\mathrm{e} 013757$.

Publisher's Note Springer Nature remains neutral with regard to jurisdictional claims in published maps and institutional affiliations.

\section{Affiliations}

\section{Agneta Månsson Broberg ${ }^{1,2}$ • Jürgen Geisler ${ }^{3}$ • Suvi Tuohinen ${ }^{4}$ - Tanja Skytta ${ }^{5}$ • Pórdís Jóna Hrafnkelsdóttir ${ }^{6}$. Kirsten Melgaard Nielsen ${ }^{7}$. Elham Hedayati ${ }^{8,9} \cdot$ Torbjørn Omland $^{10} \cdot$ Birgitte V. Offersen $^{11} \cdot$ Alexander R. Lyon $^{12}$. Geeta Gulati ${ }^{13,14}$}

1 Department of Cardiology, Karolinska University Hospital, Stockholm, Sweden

2 Department of Medicine, Huddinge, Karolinska Institutet, Stockholm, Sweden

3 Department of Oncology, Akershus University Hospital, Lørenskog \& Institute of Clinical Medicine, University of Oslo, Campus AHUS, Lørenskog, Norway

4 Heart and Lung Center, Helsinki University Hospital, Helsinki, Finland

5 Department of Oncology, Tampere University Hospital, Tampere, Finland

6 Department of Cardiology, Landspitali University Hospital, Reykjavík, Iceland and Faculty of Medicine, University of Iceland, Reykjavík, Iceland

7 Department of Cardiology, Aarhus University Hospital, Aarhus, Denmark
8 Department of Oncology-Pathology, Karolinska Institute, Stockholm, Sweden

9 Department of Breast Cancer, Sarcoma and Endocrine Tumors, Theme Cancer, Karolinska University Hospital, Stockholm, Sweden

10 Department of Cardiology, Akershus University Hospital, Lørenskog and Institute of Clinical Medicine, University of Oslo, Oslo, Norway

11 Department of Experimental Clinical Oncology \& Department of Oncology, Aarhus University Hospital, Aarhus, Denmark

12 Cardio-Oncology Service, Royal Brompton Hospital and Imperial College London, London, UK

13 Department of Cardiology, Oslo University Hospital, Postbox 4950, Ullevål, Nydalen, 0424 Oslo, Norway

14 Department of Research, Akershus University Hospital, Lørenskog and Institute of Clinical Medicine, University of Oslo, Oslo, Norway 